\title{
Partial Densities of States, Scattering Matrices, and Green's Functions
}

\author{
V. Gasparian*, T. Christen, and M. Büttiker \\ Département de physique théorique, Université de Genève, 24 Quai Ernest-Ansermet \\ CH-1211 Genève, Switzerland
}

\begin{abstract}
The response of an arbitrary scattering problem to quasi-static perturbations in the scattering potential is naturally expressed in terms of a set of local partial densities of states and a set of sensitivities each associated with one element of the scattering matrix. We define the local partial densities of states and the sensitivities in terms of functional derivatives of the scattering matrix and discuss their relation to the Green's function. Certain combinations of the local partial densities of states represent the injectivity of a scattering channel into the system and the emissivity into a scattering channel. It is shown that the injectivities and emissivities are simply related to the absolute square of the scattering wave-function. We discuss also the connection of the partial densities of states and the sensitivities to characteristic times. We apply these concepts to a $\delta$ barrier and to the local Larmor clock.
\end{abstract}

PACS numbers: $72.10 . \mathrm{Bg}, 03.80 .+\mathrm{r}$

\section{INTRODUCTION}

Densities of states (DOS) play an important role in a number of different physical contexts. For example, thermodynamic properties, tunneling spectroscopy, electrical conduction phenomena, and charging effects depend strongly on the DOS of the respective system under consideration. In recent works on ac transport in mesoscopic conductors [1]:2] it was found that many results can be expressed in a very transparent way if the concept of the DOS is generalized. In particular, it was shown that it is not only the total DOS but also parts of it which have physical significance. In this work we point to the generality of this decomposition of the total DOS and present expressions for partial densities of states (PDOS) in terms of the scattering matrix, the Green's function, and the absolute square of scattering wave-functions. Decompositions of the total DOS into partial DOS appear naturally in scattering problems in which one is concerned with the response of the system to a small perturbation $\delta U(x)$ of the potential $U(x)$. An example is the mentioned self-consistent treatment of electrical ac-transport in mesoscopic conductors. Other examples are the Larmor clock [3 8] and the optical clock [9], where the tunneling of electrons and photons, respectively, through a barrier containing a magnetic field is investigated. It turns out that the PDOS determine the rotation of the spin polarization and the Faraday rotation, respectively.

A one-dimensional scattering problem is characterized by a scattering matrix with elements $s_{\alpha \beta}$. The indices $\alpha$ and $\beta$ label out-going and incoming scattering channels, respectively, of the system under consideration. For the two-channel case as discussed below, these indices take the values 1 and 2 to designate reference points $x_{1}$ and $x_{2}$ at the left and the right side of the scattering region, respectively. The absolute squares of the scattering matrix elements determine the transmission probability $T=\left|s_{21}\right|^{2}=\left|s_{12}\right|^{2}$ and the reflection probability $R=1-T=\left|s_{11}\right|^{2}=\left|s_{22}\right|^{2}$. The response of the system can be characterized by a set of local PDOS, $d n_{\alpha \beta}(x) / d E$, and a set of sensitivities, $\eta_{\alpha \beta}(x)$, which are directly connected to the scattering-matrix element $s_{\alpha \beta}$. In general, the scattering matrix $s_{\alpha \beta}(E, U(x))$ is a function of the incident energy of the carriers and is a functional of the potential $U(x)$. To linear order in a perturbation, $\delta U(x)$, the density response and the current response of the scattering problem can be expressed with the help of the local PDOS [2]

$$
\frac{d n_{\alpha \beta}}{d E}(x) \equiv-\frac{1}{4 \pi i}\left(s_{\alpha \beta}^{\dagger} \frac{\delta s_{\alpha \beta}}{\delta U(x)}-\frac{\delta s_{\alpha \beta}^{\dagger}}{\delta U(x)} s_{\alpha \beta}\right)
$$

and with the help of the sensitivities

$$
\eta_{\alpha \beta}(x) \equiv-\frac{1}{4 \pi}\left(s_{\alpha \beta}^{\dagger} \frac{\delta s_{\alpha \beta}}{\delta U(x)}+\frac{\delta s_{\alpha \beta}^{\dagger}}{\delta U(x)} s_{\alpha \beta}\right),
$$

*Permanent address: Department of Physics, Yerevan State University, 375049 Yerevan, Armenia 
where $\delta / \delta U(x)$ denotes a functional derivative. The local PDOS represent a decomposition of the total local DOS [2]

$$
\frac{d n}{d E}(x)=\sum_{\alpha \beta} \frac{d n_{\alpha \beta}}{d E}(x) .
$$

They are based on both a pre-selection and post-selection of carriers, i.e. they group carriers according to the asymptotic region from which they arrive $(\beta)$ and according to the asymptotic region into which they are scattered $(\alpha)$. We emphasize that the PDOS are mathematical constructions. Whether these quantities are by themselves of physical relevance might well depend on the problem under investigation. While we find that the off-diagonal PDOS are positive this is not always the case for the diagonal elements. All local DOS can be obtained by summation of the local PDOS, and the global quantities associated with an entire segment or volume of the system are obtained by spatial integration.

It is the purpose of this work to present a discussion of the PDOS and to relate them to the Green's function, to dwell times, and to scattering wave-functions. The paper is organized as follows. The scattering problem to be considered is introduced in Sect. III. Some well-known results concerning the local DOS in terms of the Green's function and in terms of the scattering matrix are recalled in Sects. III and IV, respectively. Section $\mathrm{V}$ provides the relation between the basic PDOS (11) and the Green's function. In Sect. VI, we encounter decompositions of the local DOS on a next higher level, based only on a pre-selection or based only on a post-selection. We call the local PDOS which is generated by carriers incident from the asymptotic region $\alpha$ regardless into which region the carriers are finally scattered the injectivity [10] of channel $\alpha$ :

$$
\frac{d \bar{n}_{\alpha}}{d E}(x) \equiv \sum_{\beta} \frac{d n_{\beta \alpha}}{d E}(x) .
$$

The decomposition of the local DOS into injectivities represents thus a pre-selection. Similarly, we can ask about a decomposition of the local DOS into emissivities according to the asymptotic region into which carriers are scattered regardless of the channel through which the carriers entered the scattering region. The emissivity into channel $\alpha$ is given by

$$
\frac{d \underline{n}_{\alpha}}{d E}(x)=\sum_{\beta} \frac{d n_{\alpha \beta}}{d E}(x) .
$$

The decomposition of the local DOS into emissivities represents thus a post-selection.

In Sect. VII we relate the injectivity (and the emissivity) to the time a particle dwells in a narrow region. The dwell time [5] is in turn connected to the absolute square of the scattering wave-function. For instance, Eq. (4) has a simple interpretation in terms of the time a carrier is dwelling in an interval $d x$ regardless of where it is finally scattered. In terms of the scattering wave-function $\Psi_{\alpha, B}(x)$ which has unit incident amplitude in the region $\alpha$ in the presence of a uniform magnetic field $B$, the dwell time of a particle in an interval $d x$ at the point $x$ is $d \tau_{\alpha, B}=d x\left|\Psi_{\alpha, B}\right|^{2} / J$ where $J$ is the incoming current carried by the state $\Psi_{\alpha, B}$. We show that the injectivity is directly related to the dwell time and, therefore, to the wave function according to the expression

$$
d \tau_{\alpha, B}(x)=h \frac{d \bar{n}_{\alpha, B}}{d E}(x) d x=\frac{\left|\Psi_{\alpha, B}(x)\right|^{2}}{J} d x .
$$

Similarly, the emissivity is related to the square of the amplitude of the wave function $\Psi_{\alpha,-B}(x)$ calculated for a reversed magnetic field:

$$
d \tau_{\alpha,-B}(x)=h \frac{d \underline{n}_{\alpha, B}}{d E}(x) d x=\frac{\left|\Psi_{\alpha,-B}(x)\right|^{2}}{J} d x .
$$

The injectivity and the emissivity are identical in the absence of a magnetic field. A connection between the functional derivatives of the scattering matrix and the local absolute squares of the wave functions is obtained from a combination of Eqs. (1) and (6).

Section VIII is devoted to the sensitivities (2), which can be understood as local response of the transmission probabilities to a potential change. They are of great importance in a self-consistent theory of nonlinear conduction [11. Finally, we discuss in Sect. [X two examples, namely a localized impurity in a one-dimensional conduction channel and the local Larmor clock.

Before proceeding, we mention that there are some recent experimental indications for the relevance of the PDOS. 
In an impressive experiment with a quantum Hall system, Chen et al. 12] measured capacitance coefficients in a three-terminal geometry and presented an interpretation in terms of PDOS. Christen and Büttiker [13] discussed the low-frequency admittance of quantized Hall conductors with arbitrary topologies. The same authors found that the dependence of the properties of a quantum point contact on the PDOS results in steps of the capacitance and of the low-frequency admittance in synchronism with the conductance steps [14]. Leadbeater and Lambert [15] explained that an experimentally found [16] asymmetry in the STM tunneling conductance into vortices in a superconductor is due to an asymmetry in the injectivity associated with particle/hole channels.

\section{THE SCATTERING PROBLEM}

Consider the one-dimensional scattering problem sketched in Fig. 11. In a region $x_{1}<x<x_{2}$ scattering is assumed to be purely elastic. Of interest are the PDOS and the sensitivities in this region. The global scattering properties are described by the scattering-matrix elements $s_{\alpha \beta}$ which are the ratio of the current amplitudes of the out-going waves at $x_{\alpha}$ and of the incoming waves at $x_{\beta}$. Note that the scattering matrix is here defined with respect to current amplitudes at finite $x_{\alpha}(\alpha=1,2)$, and not with respect $x \rightarrow \pm \infty$. In the sequel, all quantities are absolute quantities rather than defined relatively to a free particle. For example, we deal with scattering phases rather than phase shifts and with total times rather than delay times (i.e. time differences relative to the free particle).

Scattering is due to a stationary potential $U(x)$ localized in the region $[a, b]$. To be definite, we assume $x_{1}<a<$ $0<b<x_{2}$ and that an absolute maximum of the potential, if any, is located at $x=0$. In the regions $\Omega_{1}=\left[x_{1}, a\right]$ and $\Omega_{2}=\left[b, x_{2}\right]$ the potentials are constant and take the values $U_{1}$ and $U_{2}$, respectively. Quantities associated with these regions are labeled by roman indices in contrast to the greek labels which designates the boundaries $\left(x_{\alpha}\right)$ of the system (for, e.g., an electrical conductor greek and roman labels designate contacts and regions in the conductor, respectively). This distinction is conceptually important. For example, in general the number of contacts differs from the number of relevant regions [13].

In the region $\Omega_{l}$ the wavenumber $k_{l}$ of a particle is related to the energy $E$ by $E=\left(\hbar k_{l}\right)^{2} / 2 m+U_{l}$. This energy dispersion defines in, e.g., $\Omega_{1}$ a scattering channel with a left incoming branch in which particles have the positive velocity $v_{1}=\hbar k_{1} / m$ and an out-going branch in which particles have the negative velocity $-v_{1}$. An analogous (inverse) relation holds in $\Omega_{2}$.

Below, the classical turning points $x_{L}$ and $x_{R}$ at the left and the right side of an opaque barrier, respectively, will be important. For a transparent scattering obstacle, where $\operatorname{Max}_{x}\{U(x)\}<E$, we define $x_{L}=x_{R}=0$ at the maximum of the barrier. We call the system 'large', if $x_{L}-x_{1}$ or $x_{2}-x_{R}$ is much larger then the typical wavelength $\lambda$ of the particle. The semiclassical (WKB) regime is applicable, if the characteristic scale of the space dependence of $U(x)$ is much larger then the typical wave length $\lambda$.

\section{DENSITY OF STATES AND GREEN'S FUNCTION}

We recall briefly some useful results concerning the retarded single-particle Green's function $G(x, \tilde{x})$ [17]. The scattering problem defined in the last section is associated with the Hamiltonian

$$
H=-\frac{\hbar^{2}}{2 m} \partial_{x}^{2}+U(x)
$$

The retarded Green's function is then defined as the regular solution of

$$
(E+i \epsilon-H) G(x, \tilde{x})=\delta(x-\tilde{x})
$$

where one takes the limit $\epsilon \rightarrow 0^{+}$and where $\delta(x)$ is the Dirac delta function. This Green's function can be interpreted as the quantum mechanical probability amplitude for the propagation of the particle from $\tilde{x}$ to $x$. Since we are interested only in scattering states we concentrate on the continuous part of the spectrum of $H$. Effects of the discrete part of the spectrum (belonging to localized states) on the PDOS are disregarded.

The local DOS at an energy $E$ is given by the imaginary part of the Green's function

$$
\frac{d n}{d E}(x)=-\frac{1}{\pi} \operatorname{Im}\{G(x, x)\}
$$

From the local DOS one obtains the global DOS 


$$
\frac{d N}{d E}=\int_{x_{1}}^{x_{2}} d x \frac{d n}{d E}(x)=-\int_{x_{1}}^{x_{2}} d x \frac{1}{\pi} \operatorname{Im}\{G(x, x)\}
$$

For example, the retarded Green's function of a free particle $(U(x) \equiv 0)$ with wavenumber $k=\sqrt{2 m E} / \hbar$ is given by $G(x, \tilde{x})=-i /(\hbar v) \exp (i k|x-\tilde{x}|)$ with the particle velocity $v=\hbar k / m$. Consequently, the local DOS of a ballistic channel is $2 / h v$. The DOS in a channel of length $L$ is then $2 L / h v$. This can be seen by noticing that $k L$ gives the number $N(E)$ of nodes (which count the states) of the wave function with energy $E$; the derivative with respect to energy yields the DOS.

\section{DENSITY OF STATES AND SCATTERING MATRIX}

Some useful results which relate the DOS to the scattering matrix follow already from general expressions of the scattering matrix elements in terms of the transmission and reflection probabilities and the scattering phases $\phi$ and $\phi \pm \phi_{a}$. Here $\phi$ is the total phase accumulated in a transmission event and $\phi \pm \phi_{a}$ are the phases accumulated by a particle which is incident from the left or the right and which is reflected. The phases are measured at $x_{1}$ and $x_{2}$. The scattering-matrix elements can be written in the form

$$
\mathbf{s}(E)=\left(\begin{array}{cc}
r & t \\
t & r^{\prime}
\end{array}\right)=\left(\begin{array}{cc}
-i \sqrt{R} \exp \left(i \phi+i \phi_{a}\right) & \sqrt{T} \exp (i \phi) \\
\sqrt{T} \exp (i \phi) & -i \sqrt{R} \exp \left(i \phi-i \phi_{a}\right)
\end{array}\right) .
$$

This scattering matrix is assumed to be symmetric which holds in the absence of a magnetic field. For a spatially symmetric barrier and for symmetrically located $x_{1}$ and $x_{2}$ the phase asymmetry $\phi_{a}$ vanishes and one has additionally $r=r^{\prime}$.

Avishai and Band [18] showed that the one-dimensional DOS of a large system is given by the energy derivative of the scattering phase

$$
\frac{d N}{d E}=\frac{1}{4 \pi i} \sum_{\alpha \beta}\left(s_{\alpha \beta}^{\dagger} \frac{d s_{\alpha \beta}}{d E}-\frac{d s_{\alpha \beta}^{\dagger}}{d E} s_{\alpha \beta}\right)=\frac{1}{\pi} \frac{d \phi}{d E} .
$$

In the absence of scattering the phase is given by $\phi=k L$ which again implies $d N / d E=2 L / h v$. However, the DOS of an open and finite system is not given by Eq. (13) but must be calculated by the spatial integration (11) of the local DOS. Gasparian et al. [20] calculated the integral (11) in a finite region and expressed the final results in terms of the scattering-matrix elements. The integrated DOS differs from (13) by a correction which contains the reflection amplitudes divided by the energy:

$$
\frac{d N}{d E}=\frac{1}{\pi} \frac{d \phi}{d E}+\operatorname{Im}\left\{\frac{r+r^{\prime}}{4 \pi E}\right\}=\frac{1}{\pi}\left(\frac{d \phi}{d E}-\frac{\sqrt{R}}{4 \pi E} \cos (\phi) \cos \left(\phi_{a}\right)\right) .
$$

The relative difference of the results (13) and (14) is of $\mathcal{O}(\lambda / L)$. This implies that the correction term can be neglected for large systems, for large energies, and in the semiclassical (WKB) case (and, of course, if $R$ is negligible). The local DOS can not only be obtained from Eq. (10), but alternatively also from Eq. (3), i.e. from the scattering matrix $s_{\alpha \beta}$ and its functional derivative with respect to the potential $U(x)$. In the next section we work out a relation between these two approaches in view of the PDOS.

\section{PARTIAL DENSITIES OF STATES}

The aim now is to derive simple expressions for the basic PDOS (1) by using the Green's function. Before doing this, we discuss two cases which yield some vivid insight. First, we calculate the PDOS in a large system directly from the energy derivative of the scattering matrix (12). Secondly, we construct the local PDOS in the WKB approximation from phase-space arguments. 


\section{A. Global partial densities of states in a large interval}

If we are not concerned with effects of the order of $\mathcal{O}(\lambda / L)$ the global PDOS in a large interval can be found by taking energy derivatives of the scattering matrix

$$
\frac{d N_{\alpha \beta}}{d E}=\frac{1}{4 \pi i}\left(s_{\alpha \beta}^{\dagger} \frac{d s_{\alpha \beta}}{d E}-\frac{d s_{\alpha \beta}^{\dagger}}{d E} s_{\alpha \beta}\right)
$$

Using the specific from (12) of the scattering matrix one finds

$$
\begin{aligned}
\frac{d N_{11}}{d E} & =\frac{R}{2 \pi} \frac{d\left(\phi+\phi_{a}\right)}{d E} \\
\frac{d N_{12}}{d E} & =\frac{d N_{21}}{d E}=\frac{T}{2 \pi} \frac{d \phi}{d E} \\
\frac{d N_{22}}{d E} & =\frac{R}{2 \pi} \frac{d\left(\phi-\phi_{a}\right)}{d E} .
\end{aligned}
$$

Note that the local quantities $d n_{\alpha \beta} / d E$ can be written formally by replacing the derivatives $d / d E$ in (16)-(18) by the functional derivatives $-\delta / \delta U(x)$. Clearly, the dependence of the PDOS (16)-(18) on the transmission and reflection probabilities had to be expected. The PDOS $d N_{12} / d E$ associated with particles transmitted from the right to the left is $T / 2$ times the total DOS, while $d N_{11} / d E$ must be proportional to the reflection probability. In the following paragraph such arguments are used to construct the local PDOS in the semiclassical approximation.

\section{B. Semiclassical partial densities of states}

In the semiclassical case, the local PDOS can be obtained with the help of the simple phase-space arguments of Ref. 14]. In Fig. 2 we sketched the classical phase space of the scattering problem of Fig. 1. Consider the trajectories at energy $E$ (thin curves in Fig. 2). Since the phase-space area per state corresponds to Planck's constant $h$, the semiclassical DOS is related to the energy derivative of the phase-space area $\Phi$ enclosed by the trajectories of positive and negative momentum: $d N^{(q c)} / d E=h^{-1} d \Phi / d E$ [21]. In Fig. 2, the phase-space region $d \Phi$ is indicated by a grey filling. From the classical equation of motion one obtains for the local DOS $d n^{(q c)} / d E=(2 / h v)(1-U(x) / E)^{-1 / 2}$ and $d n^{(q c)} / d E=0$ for real and imaginary momenta, respectively.

We construct now the local PDOS from the local DOS and the reflection and transmission probabilities. We mention that there exists a WKB expression for $T$ in both cases of opaque and transparent barrier [22]. We may restrict ourselves to $x_{1}<x<x_{L}$; for $x_{R}<x<x_{2}$ the results are obtained by appropriately interchanging the indices 1 and 2. Since a relative fraction $T$ of the particles with positive momentum are transmitted from $x_{1}$ to $x_{2}$ (and vice versa) one has

$$
\frac{d n_{12}^{(q c)}}{d E}(x)=\frac{d n_{21}^{(q c)}}{d E}(x)=\frac{T}{2} \frac{d n^{(q c)}}{d E}(x)
$$

A relative fraction $R$ of the particles with positive momentum and a relative fraction $1-T$ of particles with negative momentum contribute to the local PDOS of reflected particles, hence

$$
\frac{d n_{11}^{(q c)}}{d E}(x)=R \frac{d n^{(q c)}}{d E}(x) .
$$

Because on the left of the barrier there are no classical trajectories which both emanate at and return to $x_{2}$, one concludes

$$
\frac{d n_{22}^{(q c)}}{d E}(x)=0
$$

Next, we calculate the quantum mechanical corrections to these expressions. Interestingly, it turns out that for the fully quantum mechanical problem $d n_{22} / d E$ does not vanish. 


\section{Partial densities of states and the Green's function}

To derive exact expressions for the local PDOS, we start from the Fisher-Lee relation 23. between the scattering matrix and the Green's function

$$
s_{\alpha \beta}=-\delta_{\alpha \beta}+i \hbar \sqrt{v_{\alpha} v_{\beta}} G\left(x_{\alpha}, x_{\beta}\right)
$$

Insertion of Eq. (22) in Eq. (1) gives

$$
\frac{d n_{\alpha \beta}}{d E}(x)=-\frac{\hbar \sqrt{v_{\alpha} v_{\beta}}}{4 \pi}\left(s_{\alpha \beta}^{*} \frac{\delta G\left(x_{\alpha}, x_{\beta}\right)}{\delta U(x)}+\text { h.c. }\right)
$$

where the asterisk indicates complex conjugation. The functional derivative of the Green's function $\delta G / \delta U$ is calculated by adding to the Hamiltonian (8) the local potential variation $\delta U(x)=\delta U_{0} \delta\left(x-x_{0}\right)$. One finds for the variation of the Green's function $\delta G(x, \tilde{x})\left(x_{0}\right)=\delta U G\left(x, x_{0}\right) G\left(x_{0}, \tilde{x}\right)$ which implies

$$
\frac{\delta G\left(x_{\alpha}, x_{\beta}\right)}{\delta U(x)}=G\left(x_{\alpha}, x\right) G\left(x, x_{\beta}\right)
$$

Equation (23) can thus be written as

$$
\frac{d n_{\alpha \beta}}{d E}(x)=-\frac{\hbar \sqrt{v_{\alpha} v_{\beta}}}{4 \pi}\left(s_{\alpha \beta}^{*} G\left(x_{\alpha}, x\right) G\left(x, x_{\beta}\right)+\text { h.c. }\right) .
$$

This formula represents the central result of our work. Together with Eq. 22), it expresses the local PDOS fully in terms of the Green's function. For certain cases, Eq. (25) can be transformed to simpler expressions containing only $T, v_{\alpha}$, and $d n / d E$. It is shown in the appendix that the PDOS of transmitted particles is

$$
\frac{d n_{12}}{d E}(x)=\frac{d n_{21}}{d E}(x)=\frac{T}{2} \frac{d n}{d E}(x)
$$

which has the same form as the the WKB result (19). Since the sum over all local PDOS equals the local DOS it holds

$$
\frac{d n_{11}}{d E}(x)+\frac{d n_{22}}{d E}(x)=R \frac{d n}{d E}(x)
$$

Unfortunately, it is not possible in general to find expressions for $d n_{\alpha \alpha} / d E$ which are similarly simple as Eq. (26). In the flat potential regions, however, it is possible. Consider, e.g., $x \in \Omega_{1}$. The results for $\Omega_{2}$ follow from an appropriate exchange of the indices. We find for the local PDOS in $\Omega_{1}$ (see the appendix)

$$
\begin{aligned}
& \frac{d n_{11}}{d E}(x)=R \frac{d n}{d E}(x)-\frac{d n_{22}}{d E}(x) \\
& \frac{d n_{22}}{d E}(x)=\frac{T}{2}\left(\frac{2}{h v_{1}}-\frac{d n}{d E}(x)\right)
\end{aligned}
$$

The WKB results, Eqs. (19)-(21), are immediately recovered if one recalls that $d n^{(q c)} / d x=2 /\left(h v_{l}\right)$ in $\Omega_{l}$. Clearly, for vanishing transmission $T=0$ there are no states on the left side of a barrier which are scattered from $x_{2}$ to $x_{2}$, and (28) and (29) vanishes. Below we show that for finite $T$ the local PDOS (29) can be negative. Thus one concludes that, in general, the basic PDOS can not be interpreted as densities of states in the usual sense of the word.

\section{INJECTIVITY AND EMISSIVITY}

In the introduction we mentioned that in many cases the injectivity (4) and the emissivity (5) are the physically relevant PDOS. From their definition it follows that the sum over all emissivities and the sum over all injectivities is equal to the local DOS. In the absence of a magnetic field, as is the case here, injectivity and emissivity are equal to each other [2]. In principle, they must be calculated from Eq. (25). Again, they simplify considerably in the regions where the potential is uniform. From Eqs. 26)-(29) one obtains in, e.g., $\Omega_{1}$ 


$$
\begin{aligned}
& \frac{d \bar{n}_{1}}{d E}(x)=\frac{d \underline{n}_{1}}{d E}(x)=\frac{d n}{d E}(x)-\frac{T}{h v_{1}} \\
& \frac{d \bar{n}_{2}}{d E}(x)=\frac{d \underline{n}_{2}}{d E}(x)=\frac{T}{h v_{1}} .
\end{aligned}
$$

The injectivity $d \bar{n}_{2} / d E$ is constant in $\Omega_{1}$. This follows from the fact that the injectivity is proportional to the absolute square of the scattering wave-function which is shown in the next section.

The injectances and emittances of a large system can be expressed by energy derivatives of the scattering-matrix elements

$$
\begin{aligned}
& \frac{d \bar{N}_{1}}{d E}=\frac{d \underline{N}_{1}}{d E}=\frac{1}{2 \pi}\left(\frac{d \phi}{d E}+R \frac{d \phi_{a}}{d E}\right) \\
& \frac{d \bar{N}_{2}}{d E}=\frac{d \underline{N}_{2}}{d E}=\frac{1}{2 \pi}\left(\frac{d \phi}{d E}-R \frac{d \phi_{a}}{d E}\right) .
\end{aligned}
$$

The injectance (and emittance) contains the reflected part of the DOS associated with the phase asymmetry. In the following section we show that the injectance (and the emittance) is related to a dwell time.

\section{DWELL TIMES}

There exists a vast literature on characteristic times (e.g., traversal, reflection, and dwell times) for the motion of a particle in the presence of a barrier potential (see, e.g., Refs. 24,25] and Refs. therein). Such times are closely related to PDOS. In classical mechanics the time $\tau_{c l}$ needed by a particle to traverse a piece of a trajectory is given by the energy derivative of the phase-space area enclosed by the trajectory and the space axis. If one recalls the relation between phase-space area and DOS one finds for the time the particle resides in a given region and the semiclassical DOS a relation of the type $\tau_{c l}=h d N / d E$.

The definition of characteristic times in quantum dynamics is a more subtle undertaking. We derive now an expression for the time $d \tau_{\alpha}$ a particle injected at $x_{\alpha}$ dwells in the region $[x, x+d x]$, and we show that it is related to the injectivity $d \bar{n}_{\alpha} / d E$ at $x$ [6]11]. Assume that a particle current $J$ is injected at, e.g., $x_{1}$. The dwell time in a neighbourhood of $x_{0}$ is defined as the ratio of the particle number in the interval $\left[x_{0}, x_{0}+d x\right]$ and the incoming current:

$$
d \tau_{1}\left(x_{0}\right)=\frac{\left|\Psi\left(x_{0}\right)\right|^{2}}{J} d x
$$

Obviously, this equation describes a balance equation: the injected current equals the decay rate of the probability in $\left[x_{0}, x_{0}+d x\right]$. To calculate the dwell time, we follow closely Ref. [6] and introduce an infinitesimal particle absorption of strength $d \Gamma$ at $x_{0}$. This absorption is described by an imaginary perturbation $d H=-i(\hbar / 2) \delta\left(x-x_{0}\right) d \Gamma$ of the Hamiltonian (8). The perturbed Hamiltonian is not hermitian which implies that the continuity equation for the quantum mechanical probability density obtains a sink term at $x_{0}$ :

$$
\partial_{t}|\Psi(x)|^{2}+\partial_{x} j=-d \Gamma|\Psi(x)|^{2} \delta\left(x-x_{0}\right)
$$

Here, $j$ is the usual quantum mechanical current density. The current of absorbed particles is $d j=-d \Gamma\left|\Psi\left(x_{0}\right)\right|^{2}$. Thus, the infinitesimal dwell time can be written as $d \tau_{1}=-d x(d j / d \Gamma) / J$. Now we use $d j=J(d R+d T)$, where $d R$ and $d T$ are the variations of the reflection and the transmission probabilities, $R=\left|s_{11}\right|^{2}$ and $T=\left|s_{21}\right|^{2}$, respectively, for particles coming from the left. These variations are obtained from an expansion of the scattering matrix elements, $s_{\alpha 1}=$ $s_{\alpha 1}^{(0)}+\left(\delta s_{\alpha 1} / \delta U\right) d H$. With the help of Eq. (11) one can write $d R=-h d \Gamma\left(d n_{11}\left(x_{0}\right) / d E\right)$ and $d T=-h d \Gamma\left(d n_{21}\left(x_{0}\right) / d E\right)$. The case where particles are injected at $x_{2}$ is treated analogously. Dropping the index of $x_{0}$, the dwell time in a region $[x, x+d x]$ for particles coming from $x_{\alpha}$ can be expressed in the form

$$
d \tau_{\alpha}(x)=h \frac{d \bar{n}_{\alpha}}{d E}(x) d x
$$

which is proportional to the injectivity. The dwell time $\tau_{\alpha}$ of a finite region is obtained with a spatial integration, i.e. it is essentially the injectance $d \bar{N}_{\alpha} / d E$ of this region. The specific dependence on the magnetic field stated in Eqs. (6) and (7) is a consequence of reciprocity, i.e. $d \bar{n}_{\alpha, B} / d E=d \underline{n}_{\alpha,-B} / d E$ [2]. The characteristic times associated with finite regions must be calculated by spatial integration of a density (the injectivity) and are not given simply by 
energy derivatives of phases. This was already clear in the discussion of the collision times by Smith [26] by Jauch and Marchand [27]. It has also been pointed out by Gasparian and Pollak [28] when they compared Larmor-clock times with times derived from energy derivatives. Recently, a relation between DOS and dwell times has been investigated by Iannaccone [29]. To which extent the times obtained from energy derivatives provide a reasonable approximation follows from the remark at the end of Sect. IV. In particular, for large systems this approximation can be accepted.

\section{SENSITIVITIES}

Let us next investigate the sensitivities (2) and their connection to the Green's function. We first mention that the sensitivities are simply related to the functional derivatives of the transmission probability. We have $4 \pi \eta_{12}=$ $-\delta T / \delta U(x)$. The unitarity of the scattering matrix implies immediately $\eta_{12}=\eta_{21}=-\eta_{11}=-\eta_{22} \equiv \eta$. In the present case of a two-channel scatterer the sensitivities are characterized by a single quantity $\eta$ which describes the dependence of the transmission probability on the local potential. Along the lines of Sect. V one derives (see appendix)

$$
4 \pi \eta=-\frac{\delta T}{\delta U(x)}=-2 T \operatorname{Re}\{G(x, x)\} .
$$

This result states that the real part of the diagonal elements of the Green's function is essentially the sensitivity. Together with Eq. (10), this leads to an expression for the diagonal elements of the Green's function

$$
G(x, x)=-\frac{2 \pi}{T} \eta(x)-i \pi \frac{d n}{d E}(x)=\frac{\delta}{\delta U(x)}(\ln \sqrt{T}+i \pi N) .
$$

The sensitivity plays a role which is complementary to that of the local DOS. We mention that from the knowledge of the sensitivity and the DOS, one can not only derive the diagonal elements (38) of the Green's function, but in principle also the non-diagonal elements. This follows immediately from Eq. (61) in the appendix.

\section{EXAMPLES}

In this section we present two examples. First, we consider a channel with a delta barrier which describes, e.g., a one-dimensional conductor with a localized impurity. Secondly, we discuss the local Larmor clock which turns out to be ultimately related to the local PDOS.

\section{A. The delta-barrier}

As a simple example, consider a ballistic conductor $\left(U_{1}=U_{2}=0\right)$ containing a delta-function impurity $U(x)=$ $V \delta(x)$ with $V \geq 0$. For convenience, we introduce the dimensionless quantity $w=V /(\hbar v)$, where $v$ is the particle velocity. The local PDOS can be calculated either directly by introducing a further $\delta$-potential of infinitesimal strength $\delta U$, calculating the scattering matrix from the transfer matrix, and using the definition (1)). Or it can be calculated with the help of the diagonal elements of the Green's function and using the results derived above. The diagonal elements of the retarded Green's function are given by

$$
G(x, x)=-\frac{i}{\hbar v}\left(1-\frac{w(i+w)}{1+w^{2}}(\cos 2 k x+i \sin 2 k|x|)\right),
$$

and the transmission and reflection probabilities are $T=1 /\left(1+w^{2}\right)$ and $R=w^{2} /\left(1+w^{2}\right)$, respectively. With the help of the function $f(x)=w \cos (2 k x)-\sin (2 k|x|)$ we can write the PDOS in the region $x_{1}<x<0$ in the form

$$
\begin{aligned}
& \frac{d n_{11}}{d E}(x)=\frac{R}{h v}\left(2-\frac{1}{w} \frac{1+2 w^{2}}{1+w^{2}} f(x)\right), \\
& \frac{d n_{12}}{d E}(x)=\frac{d n_{12}}{d E}(x)=\frac{T}{h v}\left(1-\frac{w}{1+w^{2}} f(x)\right), \\
& \frac{d n_{22}}{d E}(x)=\frac{T}{h v} \frac{w}{1+w^{2}} f(x) .
\end{aligned}
$$


Note that $f(x)$ contains fast Friedel-like oscillations. In particular, $d n_{22} / d E$ which vanishes in a semiclassical consideration contains oscillating quantum-mechanical correction terms. Using $w /\left(1+w^{2}\right)=\sqrt{R T}$, the injectivities (and the emissivities) can be written as

$$
\begin{aligned}
& \frac{d \bar{n}_{1}}{d E}(x)=\frac{2-T}{h v}-\frac{2}{h v} \sqrt{R T} f(x), \\
& \frac{d \bar{n}_{2}}{d E}(x)=\frac{T}{h v} .
\end{aligned}
$$

The local DOS is

$$
\frac{d n}{d E}(x)=\frac{2}{h v}(1-\sqrt{R T} f(x)) .
$$

The local PDOS $d n_{\alpha \beta} / d E$ and the local DOS $d n / d E$ are plotted in Fig. 3. Note that the local PDOS $d n_{\alpha \alpha} / d E$ associated with reflection can be negative. The injectivity $d \bar{n}_{1} / d E$ is shown in Fig. 因. This injectivity is proportional to the absolute square of the scattering wave-function and is, at the right side of the barrier, proportional to $T$ and space independent.

For a symmetric system $\left(x_{2}=-x_{1}=L / 2\right)$, the global DOS is

$$
\frac{d N}{d E}=\frac{2 L}{h v}+\frac{\sqrt{R T}}{2 \pi E}(1-\cos (k L)-w \sin (k L))
$$

A calculation of the global DOS from (13) yields a wrong result without oscillation terms. Such oscillations in the DOS and the PDOS should influence the conduction properties of sufficiently small conductors.

Using Eq. (39) one obtains for the sensitivity

$$
\eta=\frac{w T^{2}}{h v}(\cos (2 k x)+w \sin (2 k|x|))
$$

The sensitivity for this example is a strongly oscillating function, i. e. it contains only Friedel-like terms. Note that the corresponding global quantity, the spatially integrated sensitivity,

$$
\int_{-L / 2}^{+L / 2} d x \eta(x)=\frac{w T^{2}}{4 \pi E}(w+\sin (k L)-w \cos (k L))
$$

differs strongly from the result obtained from an energy derivative of $T$ which yields only the average value but not the oscillation terms. Since the sensitivity is not the density of an extensive quantity, it must be calculated by functional derivatives with respect to the potential even in a large system.

\section{B. The local Larmor clock}

The Larmor clock is a system where spin-polarized electrons are scattered by a rectangular potential barrier and an additional perpendicular magnetic field [5]. Outside the barrier the magnetic field vanishes but inside it is assumed to be constant and to point in $z$-direction. We denote the Larmor frequency by $\omega_{L}$, and the space coordinate of the particle by $y$ rather than by $x$. Consider electrons coming from the left side and with spin being initially polarized in $x$-direction. Due to a Larmor precession of the spin in the barrier, the expectation values of the spin components depend on the time the particle spends in the barrier. This motivates the introduction of characteristic times. In fact, one can formally define quantities $\tau_{x}, \tau_{y}$, and $\tau_{z}$ having the dimension of a time and being associated with the precession of each spin component $s_{x}, s_{y}$, and $s_{z}$, respectively. For small $\omega_{L}$ the quantum mechanical expectation values of the spin components of the transmitted particles are given by [5]

$$
\begin{aligned}
\left\langle s_{x}\right\rangle_{T} & =\frac{\hbar}{2}\left(1-\frac{1}{2} \omega_{L}^{2}\left(\tau_{x, T}\right)^{2}\right), \\
\left\langle s_{y}\right\rangle_{T} & =-\frac{\hbar}{2} \omega_{L} \tau_{y, T} \\
\left\langle s_{z}\right\rangle_{T} & =\frac{\hbar}{2} \omega_{L} \tau_{z, T} .
\end{aligned}
$$


The conservation of the spin length implies $\tau_{x, T}=\left(\tau_{y, T}^{2}+\tau_{z, T}^{2}\right)^{1 / 2}$, i.e. only two of the times are independent. Similarly, one can introduce times $\tau_{x, R}, \tau_{y, R}$, and $\tau_{z, R}$ for the reflected particles. Expressions for these times have been derived [5] in terms of derivatives with respect of the height of the potential barrier rather than with respect of the particle energy. We emphasize the quantities $\tau$ defined here can be negative and, therefore, do not correspond in general to a physical time, although they are called 'Larmor times'. Note that the only times which are positive per definition are $\tau_{x, T}$ and $\tau_{x, R}$. However, all of these quantities have a clear physical meaning independent of their sign.

Leavens and Aers [7] discussed a local version of the Larmor clock with an arbitrary barrier potential (as described in Sect. III) and a localized magnetic field inside the barrier. This means that $B$ is finite only in the small interval $[x, x+d x]$ with $a<x<b$. The Larmor times are now infinitesimal quantities proportional to the size $d x$ of the interval. Consider particles incident from the left. It is then convenient to introduce the following complex quantities [7]:

$$
\begin{aligned}
& d \tau_{t}=i \hbar \frac{\delta \ln (t)}{\delta U(x)} d x, \\
& d \tau_{r}=i \hbar \frac{\delta \ln (r)}{\delta U(x)} d x,
\end{aligned}
$$

where $t$ and $r$ are the transmission and the reflection amplitudes, respectively, introduced in Eq. (12). The Larmor times are related to $d \tau_{r, t}$ by $d \tau_{z, T}=-\operatorname{Im}\left\{d \tau_{t}\right\}, d \tau_{z, R}=-\operatorname{Im}\left\{d \tau_{r}\right\}, d \tau_{y, T}=\operatorname{Re}\left\{d \tau_{t}\right\}$, and $d \tau_{y, R}=\operatorname{Re}\left\{d \tau_{r}\right\}$. A short calculation yields

$$
\begin{aligned}
d \tau_{t} & =\frac{h}{T}\left(\frac{d n_{21}}{d E}(x)-i \eta_{21}\right) d x, \\
d \tau_{r} & =\frac{h}{R}\left(\frac{d n_{11}}{d E}(x)-i \eta_{11}\right) d x .
\end{aligned}
$$

As it must be [7] the dwell time (36) satisfies

$$
d \tau_{1}=T d \tau_{t}+R d \tau_{r}
$$

Furthermore, we obtain for the Larmor times

$$
\begin{aligned}
& T d \tau_{z, T}=h \eta_{21}(x) d x, \\
& R d \tau_{z, R}=h \eta_{11}(x) d x, \\
& T d \tau_{z, T}=h \frac{d n_{21}}{d E}(x) d x, \\
& R d \tau_{z, R}=h \frac{d n_{11}}{d E}(x) d x .
\end{aligned}
$$

Similar relations hold for particles coming from the right. The results (57)-(60) connect the local PDOS with physically well-defined quantities, which indicates the relevance of the PDOS.

We re-emphasize again that it is tempting to associate the basic PDOS $d n_{\alpha \beta} / d E$ with physically meaningful times characterizing the tunneling process. In fact this is done in a number of works (which do not explicitly use the terms DOS or even the notation used here). However, as we mentioned already some of the PDOS can be negative which would lead to negative times. Physical times are positive and an interpretation of the PDOS in this direction is misleading.

\section{SUMMARY}

In this work we discussed the decomposition of the local density of states into partial density of states which carry the information about the past and the future of the scattered particles. We defined the sensitivities which describe the response of the transmission probability to a variation of the potential. All these quantities were defined in terms of functional derivatives of the scattering matrix with respect to the effective single-particle potential which appears in the Schrödinger equation. We have discussed their formal relation to the Green's function. While the PDOS turn out to be connected to the imaginary part of the Green's function, the sensitivity is related to its real part. Also, their connection to characteristic times of the scattering process was investigated and, consequently, to the absolute square 
of the scattering states. Finally, we considered as simple illustrative examples a delta-barrier in a ballistic channel and the local Larmor clock.

It should be clear that the concepts introduced in this article apply not only to the two-channel situation but can be generalized to many-channel scattering problems. Furthermore, a similar point of view can very likely be developed even for problems with interaction for which the notion of an effective single-particle scattering matrix is not appropriate.

Acknowledgement This work has been supported by the Swiss National Science Foundation.

\section{APPENDIX}

We derive first the Eq. (26). The relation

$$
G(x, \tilde{x})=\sqrt{G(x, x) G(\tilde{x}, \tilde{x})} \exp \left(\int_{\min (x, \tilde{x})}^{\max (x, \tilde{x})} \frac{d z}{2 G(z, z)}\right)
$$

provided by Ref. [30] implies for $x_{\alpha}<x<x_{\beta}$ immediately $G\left(x_{\alpha}, x\right) G\left(x, x_{\beta}\right)=G\left(x_{\alpha}, x_{\beta}\right) G(x, x)$. It follows

$$
\frac{d n_{12}}{d E}(x)=i \frac{\hbar^{2} v_{1} v_{2}}{4 \pi}\left|G\left(x_{1}, x_{2}\right)\right|^{2}\left(G(x, x)-G^{*}(x, x)\right)
$$

which is equivalent to Eq. (26). Next, we prove the validity of Eqs. (28) and (29) for $x \in \Omega_{1}$. To do this we mention first a relation between the scattering matrix elements $s_{12}$ and $s_{x 2}$, where $s_{x 2}$ connects current amplitudes at $x_{2}$ and at $x$. Using the technique of transfer matrices and their relation to scattering matrices, one obtains $s_{12}=s_{x 2} \exp i k\left(x-x_{1}\right)$. Similarly, one shows that $s_{11}=s_{x x} \exp 2 i k\left(x-x_{1}\right)$ where $s_{x x}$ denotes the reflection amplitude at $x$. With the help of the Fisher-Lee relation (22) one shows

$$
\begin{aligned}
\frac{d n_{22}}{d E}(x) & =-\frac{\hbar v_{2}}{4 \pi}\left(s_{22}^{*} G^{2}\left(x_{2}, x\right)+h . c\right) \\
& =\frac{1}{4 \pi \hbar v_{1}}\left(i \sqrt{R} \exp \left(i \phi+i \phi_{a}\right) T \exp \left(-2 i k\left(x-x_{1}\right)\right)+\text { h.c. }\right) \\
& =-\frac{T}{2 h v_{1}}\left(s_{x x}+s_{x x}^{*}\right)=\frac{T}{h v_{1}}+\frac{T}{4 \pi i}\left(G(x, x)-G^{*}(x, x)\right)
\end{aligned}
$$

which yields Eq. (29). In similar way, one has

$$
\begin{aligned}
\frac{d n_{11}}{d E}(x) & =-\frac{\hbar v_{1}}{4 \pi}\left(s_{11}^{*} G^{2}\left(x_{1}, x\right)+h . c\right) \\
& =-\frac{\hbar v_{1}}{4 \pi}\left(s_{11}^{*} \frac{G^{2}\left(x_{1}, x\right) G^{2}\left(x_{2}, x\right)}{G^{2}\left(x_{2}, x\right)}+h . c\right) \\
& =-\frac{\hbar v_{1}}{4 \pi}\left(s_{11}^{*} \frac{G^{2}\left(x_{1}, x_{2}\right) G^{2}(x, x}{G^{2}\left(x_{2}, x\right)}+h . c\right) \\
& =-\frac{\hbar v_{1}}{4 \pi}\left(s_{x x}^{*} G^{2}(x, x)+h . c\right)=\frac{R+1}{2 h v_{1}}\left(s_{x x}+s_{x x}^{*}\right)
\end{aligned}
$$

which yields Eq. (28). Finally, the expression (37) for the sensitivity follows from

$$
\frac{\delta\left|s_{12}\right|}{\delta U(x)}=\hbar^{2} v_{1} v_{2}\left(\left|G\left(x_{1}, x_{2}\right)\right|^{2} G^{*}(x, x)+\text { h.c. }\right) \text {. }
$$

[1] M. Büttiker, H. Thomas, and A. Prêtre, Z. Phys. B 94, 133 (1994). 
[2] M. Büttiker, J. Phys.: Condensed Matter 5, 9361 (1993).

[3] A. I. Baz', Yad. Fiz. 4, 252 (1966) [Sov. Nucl. Phys. 4, 229 (1967)].

[4] V. F. Rybachenko, Yad. Fiz. 5, 895 (1967) [Sov. Nucl. Phys. 5, 635 (1967)].

[5] M. Büttiker, Phys. Rev. B 27, 6178 (1983).

[6] M. Büttiker, Traversal, reflection and dwell time for quantum tunneling, in 'Electronic properties of multilayers and lowdimensional semiconductors structures', edited by J.M. Chamberlain et al., Plenum Press, New York (1990).

[7] C. R. Leavens and G. C. Aers, Solid State Communications 67, 1135 (1988).

[8] A. M. Steinberg, Phys. Rev. Lett. 74, 2405 (1995).

[9] V. Gasparian, M. Ortuno, J. Ruiz, and E. Cuevas, Phys. Rev. Lett. 75, 2312 (1995).

[10] We follow mostly the standard nomenclature where local and spatially integrated quantities are named with a final syllable -ivity and -ance, respectively (e.g., emissivity and emittance).

[11] M. Büttiker and T. Christen, Basic elements of electrical conduction, in 'Transport in Semiconductor Submicron Structures', edited by B. Kramer et al., Kluwer Academic Publishers (Dordrecht, Boston, London, 1996).

[12] W. Chen, T. P. Smith, M. Büttiker, and M. Shayegan, Phys. Rev. Lett. 73, 146 (1994).

[13] T. Christen and M. Büttiker, Phys. Rev. B. 53, Jan. 15 (1996).

[14] T. Christen and M. Büttiker, unpublished.

[15] M. Leadbeater and C. J. Lambert, unpublished.

[16] I. Maggio-Aprile et al., Phys. Rev. Lett. 75, 2754 (1995).

[17] E.N. Economou, Green's Functions in Quantum Physics, Springer Series in Solid State Physics, edited by M. Cardona, P. Fulde, and H.-J. Quesser, Springer-Verlag Berlin, Heidelberg, New York (1979).

[18] Y. Avishai, and Y.B. Band, Phys. Rev. B32, 2674 (1985).

[19] R. Dashen, S. H. Ma, and H. J. Bernstein, Phys. Rev. 187, 345 (1969).

[20] V. Gasparian et al., Phys. Rev. B51, 6743 (1995).

[21] L. D. Landau and E. M. Lifschitz, A Course in Theoretical Physics, Vol. 3 (Pergamon Press, Oxford, 1977).

[22] S. C. Miller and R. M. Good, Phys. Rev. 91, 174 (1953).

[23] D.S. Fisher, and P.A. Lee, Phys. Rev. B 23, 6851 (1981).

[24] R. Landauer and T. Martin, Rev. Mod. Phys 66, 217 (1994).

[25] E. H. Hauge and J. A. Stovneng, Rev. Mod. Phys. 61, 917 (1989).

[26] F. T. Smith, Phys. Rev. 118, 349 (1960).

[27] J. M. Jauch and J.-P. Marchand, Helv. Phys. Acta 40, 217 (1967).

[28] V. Gasparian and M. Pollak, Phys. Rev. B 47, 2038 (1993).

[29] G. Iannaccone, Phys. Rev. B 51, 4727 (1995)

[30] A.G. Aronov, V.M. Gasparian, and Ute Gummich, J. Phys. Condens. Matter 3, 3023 (1991). 
FIG. 1. One-dimensional scattering problem described in the text. The dashed and dotted curves belong to Fermi-energies associated with a transparent and an opaque barrier, respectively.

FIG. 2. Classical phase-space plot of the scattering region in Fig. 1. The WKB density of states is obtained from the phase-space area $d \Phi$ (grey region) between two trajectories (thin lines) of energy difference $d E$. The dashed arrows indicate tunneling.

FIG. 3. Partial densities of states $d n_{11} / d E$ (dashed), $d n_{12} / d E$ (dotted), $d n_{22} / d E$ (dashed-dotted), and $d n / d E$ (solid) for the delta barrier with $T=0.8$.

FIG. 4. Injectivity (or absolute square of the scattering wave-function) $d \bar{n}_{1} / d E$ for the delta barrier with $T=0.8$. 


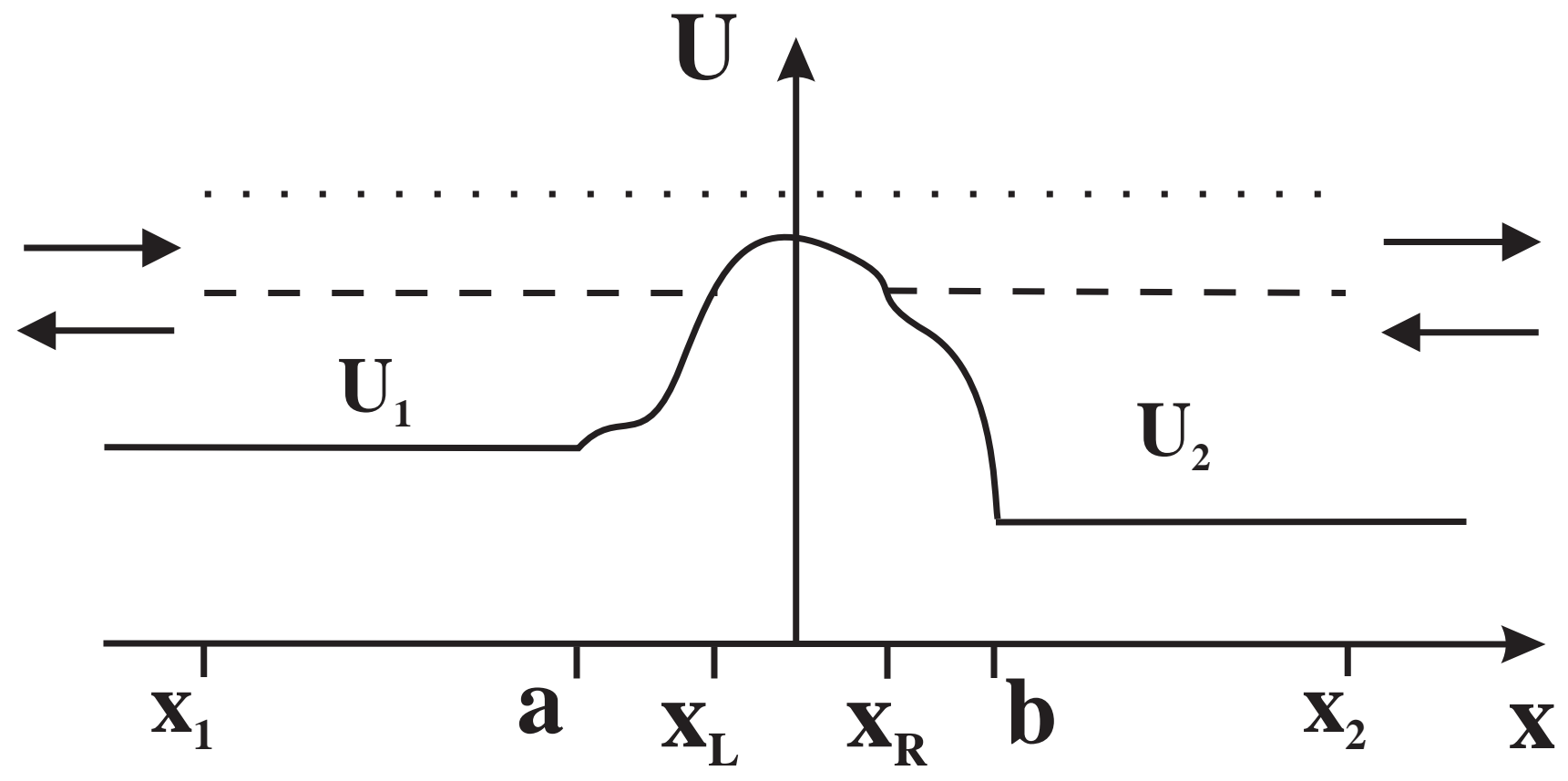




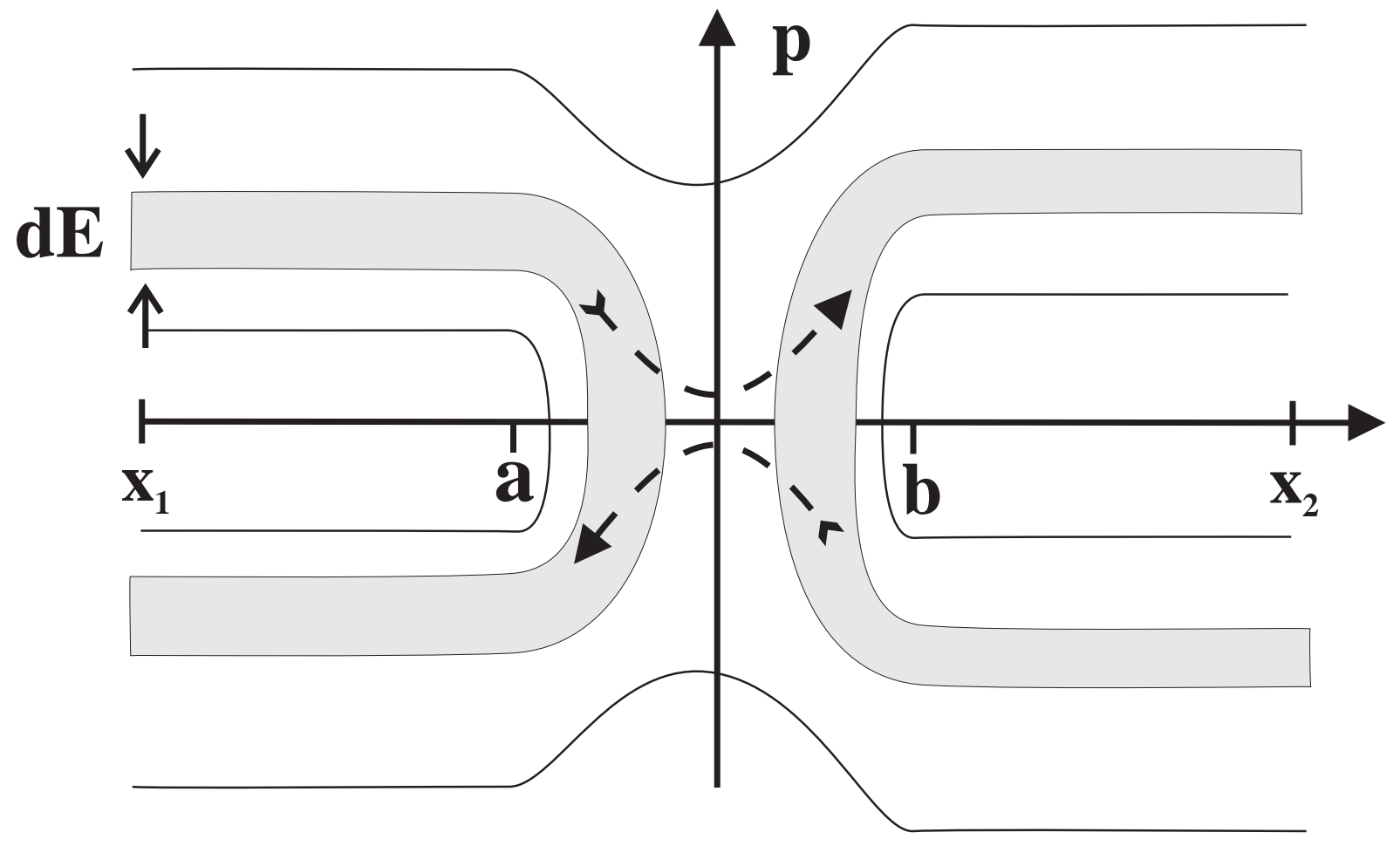




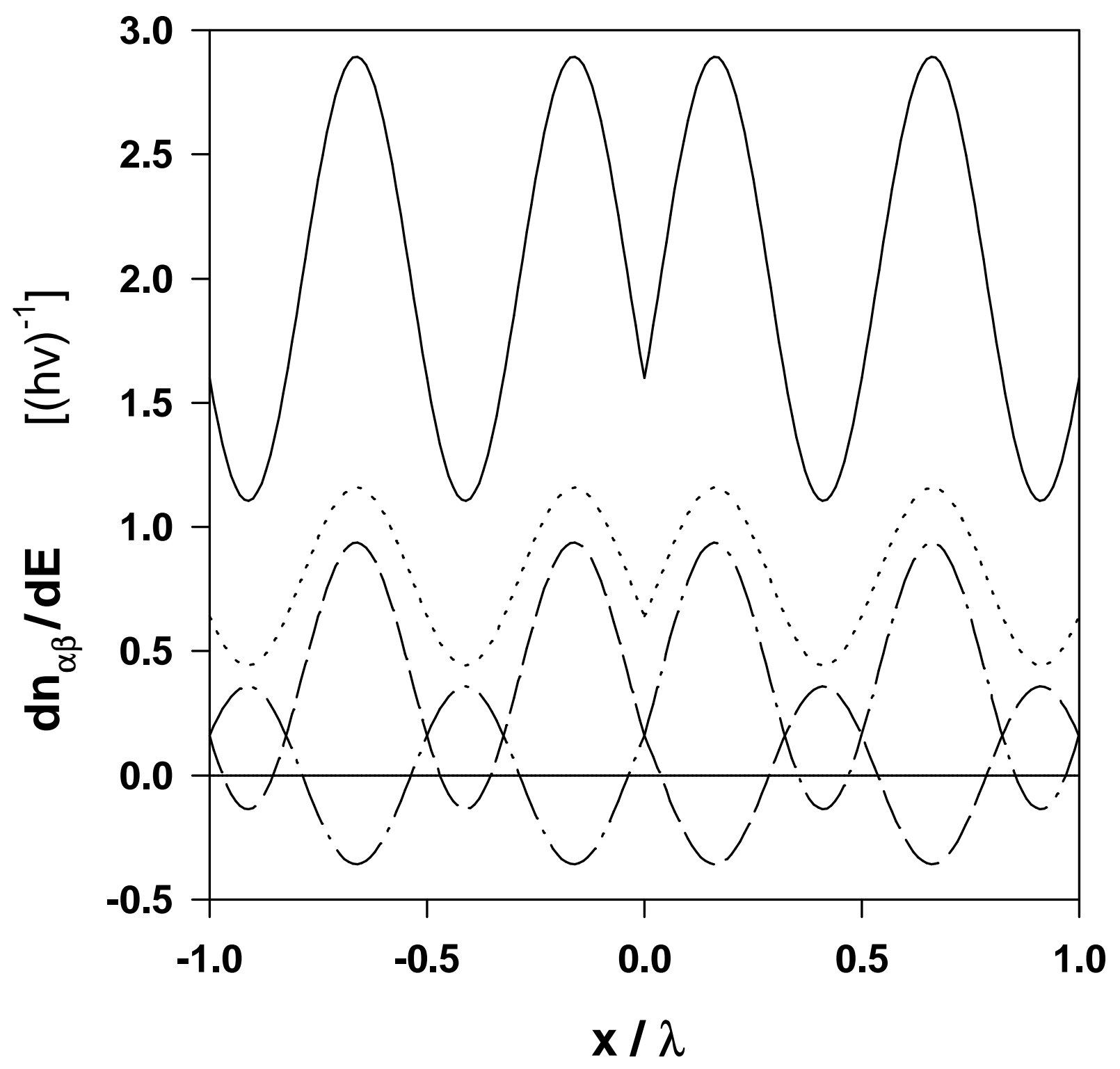




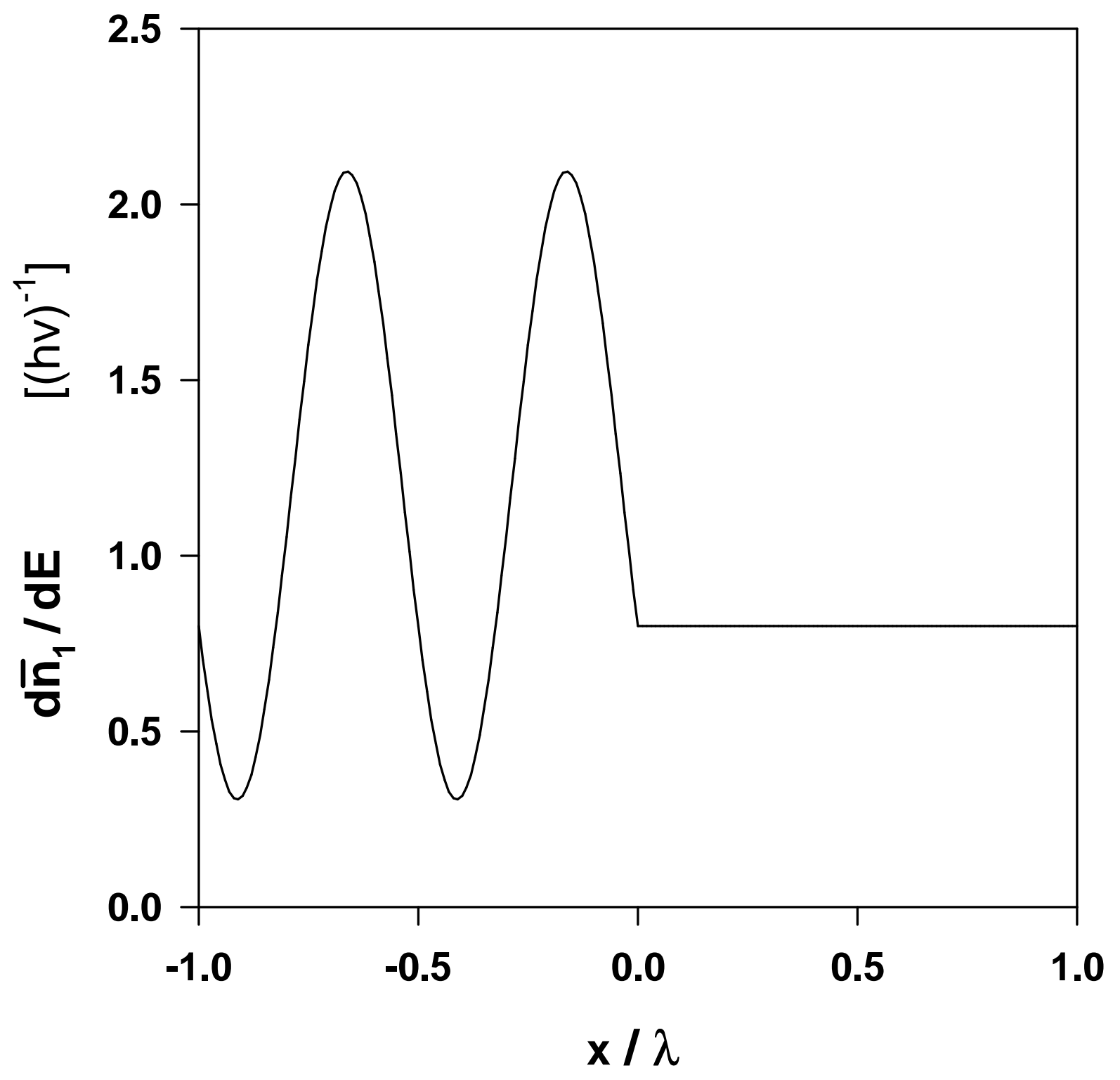

